\title{
Polarized light scattering with Paschen-Back effect, level-crossing of fine structure states and partial frequency redistribution
}

\author{
K. Sowmya ${ }^{1}$, K. N. Nagendra ${ }^{1}$, M. Sampoorna ${ }^{1}$ and J. O. Stenflo ${ }^{2,3}$ \\ ${ }^{1}$ Indian Institute of Astrophysics, Koramangala, Bengaluru, India \\ ${ }^{2}$ Institute of Astronomy, ETH Zurich, CH-8093 Zurich, Switzerland \\ ${ }^{3}$ Istituto Ricerche Solari Locarno, Via Patocchi, 6605 Locarno-Monti, Switzerland \\ ksowmya@iiap.res.in; knn@iiap.res.in; sampoorna@iiap.res.in; stenflo@astro.phys.ethz.ch
}

\begin{abstract}
The quantum interference between the fine structure states of an atom modifies the shapes of the emergent Stokes profiles in the Second Solar Spectrum. This phenomenon has been studied in great detail both in the presence and absence of magnetic fields. By assuming a flat-spectrum for the incident radiation, the signatures of this effect have been explored for arbitrary field strengths. Even though the theory which takes into account the frequency dependence of the incident radiation is well developed, it is restricted to the regime in which the magnetic splitting is much smaller than the fine structure splitting. In the present paper, we carry out a generalization of our scattering matrix formalism including the effects of partial frequency redistribution (PRD) for arbitrary magnetic fields. We test the formalism using available benchmarks for special cases. In particular we apply it to the $\mathrm{Li}$ I $6708 \AA \mathrm{D}_{1}$ and $\mathrm{D}_{2}$ line system, for which observable effects from the Paschen-Back regime are expected in the Sun's spectrum.
\end{abstract}

Subject headings: atomic processes - line: profiles - scattering - polarization - magnetic fields

\section{Introduction}

The interaction of the spin of the electrons with their orbital angular momenta leads to splitting of the atomic levels into fine structure states that are labeled by the total electronic angular momentum quantum numbers $J$. The magnetic substates belonging to these $J$ states are degenerate in the absence of a magnetic field. When a magnetic field is applied, the degeneracy is lifted and the energies of these magnetic substates are modified. With an increase in the field strength, the magnetic substates belonging to different $J$ states of a given term begin to overlap leading to a mixing of the $J$ states and $J$ no longer remains a good quantum number. The Paschen-Back effect (PBE) occurs when the splitting produced by the magnetic field is comparable to the fine structure splitting.

In the present paper we address the problem of $\mathrm{PBE}$ on a two-term atom taking into account the effects of partial frequency redistribution (PRD).
In other words, we study the $J$-state interference phenomenon in the presence of a magnetic field of arbitrary strength. In particular we derive the PRD matrix for the problem at hand and present the results obtained for the single scattering case.

Bommier (1980) developed a density matrix formalism to handle $J$-state interference in the presence of a magnetic field of arbitrary strength (including both the Zeeman and the Paschen-Back regimes). Her formalism was limited to the complete frequency redistribution (CRD) in scattering. A quantum theory of $J$-state interference phenomenon for the case of frequency coherent scattering was formulated by Stenflo (1980, 1994, 1997). Under the flat-spectrum approximation, Landi Degl'Innocenti \& Landolfi (2004, hereafter LL04) developed a QED theory for the $J$-state interference phenomenon in a multi-term atom and in the presence of magnetic fields of arbitrary strengths. Assuming CRD, Casini \& Manso Sainz (2005) considered the problem of PBE in a multi- 
term atom involving the interferences among both $J$ and $F$ states. In the linear Zeeman regime, where the fine structure splitting is larger than the splitting produced by the magnetic field, Smitha et al. (2011, 2013) developed a theory for interference between the fine structure states taking into account the effects of PRD. In the present paper, we generalize the collisionless redistribution matrix (hereafter RM) derived by Smitha et al. (2011) to include the PBE. In other words, we present a general form of the RM which holds good in Hanle, Zeeman as well as PB regimes.

$\mathrm{PBE}$ is of great interest to the stellar as well as the solar community because it serves as an effective tool to diagnose vector magnetic fields. The emergent spectral line polarization depends sensitively on the magnetic field. PBE in molecules has proven to be a good diagnostic tool in recent years for magnetic field measurements. Since the fine structure splittings in molecules are smaller than those for atoms, the PBE becomes operative for relatively lower field strengths in molecules. Attempts have been made to develop a theoretical framework for this problem and to identify and understand the signatures of this effect in the emergent line polarization (see for e.g., Asensio Ramos \& Truillo Bueno 2006; Berdvugina et al. 2005, 2006a, b; Shapiro et al. 2006, 2007). The important step is to set up the Hamiltonian in the right form and diagonalize it to get the energy eigenvalues and eigenvectors which can be used later in the computations of the Stokes parameters. To this end, in our work concerned with PBE in atoms, we use the diagonalization code of Landi Degl'Innocenti (1978). This is a computer program written for the simultaneous diagonalization of the magnetic and the hyperfine structure Hamiltonian. We modify this program suitably for the problem at hand.

In the work on $\mathrm{PBE}$ on hyperfine structure states of a two-level atom (see Sowmva et al. 2014), we derived the PRD matrix for this process. Further, we studied the characteristics of the $\mathrm{RM}$ in a single $90^{\circ}$ scattering event. The same framework can also be developed for the case of PBE on fine structure states with the straightforward replacement of the quantum numbers which we discuss in Section 3 . In Section 2 we set up the total Hamiltonian for PBE in a two-term atom. The Hamiltonian in this case has non-zero non- diagonal elements which represent the mixing of the $J$ states. The general form of the RM in terms of the irreducible spherical tensors, derived assuming the lower levels to be unpolarized and infinitely sharp, is presented in Section [3. The results for the single scattering case are discussed in Section 4 considering the fine structure states of the lithium $\mathrm{D}_{1}$ and $\mathrm{D}_{2}$ lines as an example. In the solar case, the Li I $6708 \AA$ doublet, which has the same fine structure configuration as the $\mathrm{D}_{1}$ and $\mathrm{D}_{2}$ lines of $\mathrm{NaI}$ and $\mathrm{BaII}$, but for which the fine structure splitting is only $0.15 \AA$, serves as a good candidate for application of the theory developed in the present paper. Spectropolarimetric observations of this Li I doublet have been published in Stenflo (2011). The theoretical work on the same spectral line system has been presented by Belluzzi et al. (2009) in the limit of microturbulent fields and for the non-magnetic case. Section 5 is devoted for concluding remarks.

\section{PBE in a two-term atom}

We consider a two-term atom described by the $L-S$ coupling scheme. Under the $L-S$ coupling approximation, the fine structure Hamiltonian is given by

$$
\mathcal{H}_{\mathrm{fs}}=\zeta\left(L_{k} S\right) \boldsymbol{L}_{k} \cdot \boldsymbol{S}
$$

where $\zeta\left(L_{k} S\right)$ has the dimensions of energy and is given by the 'Landé-interval' rule as

$$
\zeta\left(L_{k} S\right)=\frac{E\left(J_{k}\right)-E\left(J_{k}-1\right)}{J_{k}}
$$

Here $k=a$ (lower term) or $b$ (upper term). The energy shift due to spin-orbit coupling can be obtained from the Hund's rule 3 as

$$
\begin{aligned}
& E_{L_{k} S}\left(J_{k}\right)=\frac{1}{2} \zeta\left(L_{k} S\right) \\
\times & {\left[J_{k}\left(J_{k}+1\right)-L_{k}\left(L_{k}+1\right)-S(S+1)\right] . }
\end{aligned}
$$

If an external magnetic field is applied then its interaction with the atomic system is described by the Hamiltonian

$$
\mathcal{H}_{B}=\mu_{0}\left(\boldsymbol{L}_{k}+2 \boldsymbol{S}\right) \cdot \boldsymbol{B}
$$

where $\mu_{0}$ is the Bohr-magneton. If the applied magnetic field produces a splitting comparable to the fine structure splitting, then the magnetic 
Hamiltonian can no longer be treated as a perturbation to the spin-orbit Hamiltonian, $\mathcal{H}_{\mathrm{fs}}$. In this case the energy levels have to be found by diagonalizing the total Hamiltonian $\mathcal{H}$ given by

$$
\mathcal{H}=\mathcal{H}_{\mathrm{fs}}+\mathcal{H}_{B} .
$$

The quantization axis (z-axis) is taken to be along the applied magnetic field so that the total Hamiltonian can be diagonalized in the energy eigenvector basis $\left|L_{k} S J_{k} \mu_{k}\right\rangle$. However in the $\mathrm{PB}$ regime the magnetic field produces a mixing of the $J$ states belonging to a given term. Thus the eigenvectors of the total Hamiltonian are of the form

$$
\left|L_{k} S j_{k} \mu_{k}\right\rangle=\sum_{J_{k}} C_{J_{k}}^{j_{k}}\left(L_{k} S, \mu_{k}\right)\left|L_{k} S J_{k} \mu_{k}\right\rangle,
$$

where the symbol $j_{k}$ labels different states spanned by the quantum numbers $\left(L_{k}, S, \mu_{k}\right)$ and

$C_{J_{k}}^{j_{k}}\left(L_{k} S, \mu_{k}\right)$ are the expansion coefficients. To determine the eigenvectors $\left|L_{k} S j_{k} \mu_{k}\right\rangle$ and the corresponding eigenvalues we have to diagonalize a set of matrices of the form

$$
\left\langle L_{k} S J_{k} \mu_{k}\left|\mathcal{H}_{\mathrm{fs}}+\mathcal{H}_{B}\right| L_{k} S J_{k^{\prime}} \mu_{k}\right\rangle .
$$

The above expression indicates that a given $\mu_{k}$ can be assigned to both $J_{k}$ and $J_{k^{\prime}}$ as a result of level interference. Since the spin orbit Hamiltonian is diagonal in $J_{k}$ we have

$$
\left\langle L_{k} S J_{k} \mu_{k}\left|\mathcal{H}_{\mathrm{fs}}\right| L_{k} S J_{k} \mu_{k}\right\rangle=E_{L_{k} S}\left(J_{k}\right),(8)
$$

where $E_{L_{k} S}\left(J_{k}\right)$ is given by Equation (3). The magnetic Hamiltonian can be written in the energy eigenvector basis as

$$
\begin{aligned}
& \left\langle L_{k} S J_{k} \mu_{k}\left|\mathcal{H}_{B}\right| L_{k} S J_{k^{\prime}} \mu_{k^{\prime}}\right\rangle=\delta_{\mu_{k} \mu_{k^{\prime}}} \mu_{0} B \\
& \times\left[\mu_{k} \delta_{J_{k} J_{k^{\prime}}}+(-1)^{J_{k}+J_{k^{\prime}}+L_{k}+S+\mu_{k}}\right. \\
& \times \sqrt{\left(2 J_{k}+1\right)\left(2 J_{k^{\prime}}+1\right) S(S+1)(2 S+1)} \\
& \left.\times\left(\begin{array}{ccc}
J_{k} & J_{k^{\prime}} & 1 \\
-\mu_{k} & \mu_{k} & 0
\end{array}\right)\left\{\begin{array}{ccc}
J_{k} & J_{k^{\prime}} & 1 \\
S & S & L_{k}
\end{array}\right\}\right]
\end{aligned}
$$

The diagonalization of the total Hamiltonian gives the energy eigenvalues and the energy eigenvectors (see Landi Degl'Innocenti 1978). For simplicity we consider the PBE only in the upper term and neglect the crossing of magnetic substates belonging to different fine structure states in the lower term.

\section{PRD matrix for the Paschen-Back ef- fect on fine structure states}

The steps followed in deriving the $\mathrm{RM}$ are the same as those in the case of PBE in hyperfine structure states (see Sowmva et al. 2014). The resulting RM for $J$-state interference in the presence of magnetic fields of arbitrary strengths can also be obtained from the corresponding RM for the $F$-state interference phenomenon by the following quantum number replacement:

$$
F \rightarrow J ; \quad J \rightarrow L ; \quad I_{s} \rightarrow S ; \quad i \rightarrow j,
$$

in the latter RM. Here $F\left(=\boldsymbol{J}+\boldsymbol{I}_{\boldsymbol{s}}\right)$ is the total angular momentum, $J(=\boldsymbol{L}+\boldsymbol{S})$ is the total electronic angular momentum, $L$ is the orbital angular momentum, $I_{s}$ is the nuclear spin and $S$ is the electron spin angular momentum. $i$ and $j$ label different states spanned by the quantum numbers $\left(J, I_{s}, \mu_{F}\right)$ and $\left(L, S, \mu_{J}\right)$ respectively. Here $\mu_{F}$ and $\mu_{J}$ are the projections of $F$ and $J$ on the quantization axis. Thus the RM for $J$-state interference in the presence of a magnetic field of arbitrary strength can be written as

$$
\begin{aligned}
& \mathbf{R}_{i j}^{\mathrm{II}}\left(x, \boldsymbol{n}, x^{\prime}, \boldsymbol{n}^{\prime} ; \boldsymbol{B}\right)=\frac{3\left(2 L_{b}+1\right)}{(2 S+1)} \\
& \times \sum_{K K^{\prime} Q} \sum_{j_{a} \mu_{a} j_{f}} \sum_{\mu_{f} j_{b} \mu_{b} j_{b^{\prime}} \mu_{b^{\prime}}} \\
& \times \sum_{J_{a} J_{a^{\prime}} J_{f} J_{f^{\prime}} J_{b} J_{b^{\prime}} J_{b^{\prime \prime}}} \sum_{J_{b^{\prime \prime \prime}}}(-1)^{q-q^{\prime} q^{\prime \prime \prime} q^{\prime \prime \prime}}+Q \\
& \times \sqrt{(2 K+1)\left(2 K^{\prime}+1\right)} \cos \beta_{j_{b^{\prime}} \mu_{b^{\prime}} j_{b} \mu_{b}} \mathrm{e}^{\mathrm{i} \beta_{j_{b^{\prime}} \mu_{b^{\prime}} j_{b} \mu_{b}}} \\
& \times\left[\left(h_{j_{b} \mu_{b}, j_{b^{\prime}} \mu_{b^{\prime}}}^{\mathrm{II}}\right)_{j_{a} \mu_{a} j_{f} \mu_{f}}+\mathrm{i}\left(f_{j_{b} \mu_{b}, j_{b^{\prime}} \mu_{b^{\prime}}}^{\mathrm{II}}\right)_{j_{a} \mu_{a} j_{f} \mu_{f}}\right] \\
& \times C_{J_{f}}^{j_{f}}\left(L_{a} S, \mu_{f}\right) C_{J_{a}}^{j_{a}}\left(L_{a} S, \mu_{a}\right) C_{J_{b}}^{j_{b}}\left(L_{b} S, \mu_{b}\right) \\
& \times C_{J_{b^{\prime \prime}}}^{j_{b}}\left(L_{b} S, \mu_{b}\right) C_{J_{f^{\prime}}}^{j_{f}}\left(L_{a} S, \mu_{f}\right) C_{J_{a^{\prime}}}^{j_{a}}\left(L_{a} S, \mu_{a}\right) \\
& \times C_{J_{b^{\prime}}}^{j_{b^{\prime}}}\left(L_{b} S, \mu_{b^{\prime}}\right) C_{J_{b^{\prime \prime \prime}}}^{j_{b^{\prime}}}\left(L_{b} S, \mu_{b^{\prime}}\right) \\
& \times \sqrt{\left(2 J_{a}+1\right)\left(2 J_{a^{\prime}}+1\right)\left(2 J_{f}+1\right)\left(2 J_{f^{\prime}}+1\right)} \\
& \times \sqrt{\left(2 J_{b}+1\right)\left(2 J_{b^{\prime}}+1\right)\left(2 J_{b^{\prime \prime}}+1\right)\left(2 J_{b^{\prime \prime \prime}}+1\right)} \\
& \times\left(\begin{array}{ccc}
J_{b} & J_{f} & 1 \\
-\mu_{b} & \mu_{f} & -q
\end{array}\right)\left(\begin{array}{ccc}
J_{b^{\prime}} & J_{f^{\prime}} & 1 \\
-\mu_{b^{\prime}} & \mu_{f} & -q^{\prime}
\end{array}\right) \\
& \times\left(\begin{array}{ccc}
J_{b^{\prime \prime}} & J_{a} & 1 \\
-\mu_{b} & \mu_{a} & -q^{\prime \prime}
\end{array}\right)\left(\begin{array}{ccc}
J_{b^{\prime \prime \prime}} & J_{a^{\prime}} & 1 \\
-\mu_{b^{\prime}} & \mu_{a} & -q^{\prime \prime \prime}
\end{array}\right) \\
& \times\left(\begin{array}{ccc}
1 & 1 & K \\
q & -q^{\prime} & -Q
\end{array}\right)\left(\begin{array}{ccc}
1 & 1 & K^{\prime} \\
q^{\prime \prime \prime} & -q^{\prime \prime} & Q
\end{array}\right)
\end{aligned}
$$




$$
\begin{aligned}
& \times\left\{\begin{array}{ccc}
L_{a} & L_{b} & 1 \\
J_{b} & J_{f} & S
\end{array}\right\}\left\{\begin{array}{ccc}
L_{a} & L_{b} & 1 \\
J_{b^{\prime}} & J_{f^{\prime}} & S
\end{array}\right\} \\
& \times\left\{\begin{array}{ccc}
L_{a} & L_{b} & 1 \\
J_{b^{\prime \prime}} & J_{a} & S
\end{array}\right\}\left\{\begin{array}{ccc}
L_{a} & L_{b} & 1 \\
J_{b^{\prime \prime \prime}} & J_{a^{\prime}} & S
\end{array}\right\} \\
& \times(-1)^{Q} \mathcal{T}_{-Q}^{K}(i, \boldsymbol{n}) \mathcal{T}_{Q}^{K^{\prime}}\left(j, \boldsymbol{n}^{\prime}\right) .
\end{aligned}
$$

The assumptions underlying the derivation of Equation (11) are that the lower levels are unpolarized and infinitely sharp. See Sowmya et al. (2014) for details on the terminology and the derivation.

\section{Single scattering polarization with PBE}

As an example to study the PBE in fine structure states we consider the $L=0$ and $L=1$ terms of the two stable isotopes of neutral lithium, namely ${ }^{7} \mathrm{Li}$ and ${ }^{6} \mathrm{Li}$. The isotopic shifts are measured with respect to the reference isotope ${ }^{7} \mathrm{Li}$. In our calculations we use the isotopic shift values given in Table 1 of Belluzzi et al. (2009). The abundances for the two isotopes are also read from the same table. The total electron spin, $S=1 / 2$. The coupling between $\boldsymbol{L}$ and $\boldsymbol{S}$ results in $J=3 / 2$ and $1 / 2$ for the $L=1$ term and $J=1 / 2$ for the $L=0$ term. The transitions between these $J$ states in the absence of magnetic fields results in the $\mathrm{D}_{1}$ and $\mathrm{D}_{2}$ lines (obeying the selection rules $\Delta S=0, \Delta J=0, \pm 1)$. The wavelengths of these transitions are listed in Table 1. In the presence of a magnetic field, the non-degenerate magnetic substates give rise to 10 allowed transitions (according to the selection rule $\Delta \mu=0, \pm 1$ ) in each of the two isotopes. Among these 10 transitions, 6 are between the magnetic substates of the upper $J=3 / 2$ and the lower $J=1 / 2$ states and the rest are between those of the upper $J=1 / 2$ and the lower $J=1 / 2$ states. These transitions can be classified into three groups: $\sigma_{\mathrm{r}}(\Delta \mu=-1)$, $\pi(\Delta \mu=0)$, and $\sigma_{\mathrm{b}}(\Delta \mu=+1)$. Note that $\Delta \mu=\mu_{b}-\mu_{a}$ where $\mu_{b}$ are the magnetic substates of the upper $J$ state and $\mu_{a}$ are the magnetic substates of the lower $J$ state. The magnetic ( $\pi$ and $\sigma$ ) components of the $\mathrm{D}_{1}$ lines will be denoted with a prime in the following discussions for the sake of clarity and distinction. As per this classification, the $\mathrm{D}_{2}$ line gives rise to two $\sigma_{\mathrm{r}}$, two $\pi$, and two $\sigma_{\mathrm{b}}$ components while the $\mathrm{D}_{1}$ line gives rise to one $\sigma_{\mathrm{r}}^{\prime}$, two $\pi^{\prime}$, and one $\sigma_{\mathrm{b}}^{\prime}$ components, in each of the two isotopes. These are tabulated in Table 2, The magnetic components of the two isotopes will be distinguished by their mass numbers indicated in the superscripts to the $\pi$ and $\sigma$ components. For the computation of the Stokes profiles presented in Figures 24 we assume that an unpolarized radiation is incident on the atom at an angle $\cos \theta^{\prime}=1$ and gets scattered in a direction $\cos \theta=0$, where $\theta^{\prime}$ and $\theta$ are the colatitudes. The values of the azimuths $\chi$ and $\chi^{\prime}$ for scattered and incident rays, respectively, are assumed to be zero in this single $90^{\circ}$ scattering event. The scattered ray so obtained is given by the first column of the RM, which is then integrated over the incoming frequencies to get the singly scattered Stokes profiles. For the Li I D line system, the Stokes parameters are obtained by linearly combining the Stokes parameters computed for the individual isotopes weighted by their respective abundances. Such a linear superposition is allowed because the lines are optically thin. Following Belluzzi et al. (2009) we use a Doppler width of $60 \mathrm{~m} \AA$ for all the components.

\begin{tabular}{|c|c|c|c|}
\hline Isotope & Line & $\lambda(\AA)$ & $A\left(\mathrm{~s}^{-1}\right)$ \\
\hline${ }^{6} \mathrm{Li}$ & $\mathrm{D}_{1}$ & 6708.05534 & $3.689 \times 10^{7}$ \\
${ }^{6} \mathrm{Li}$ & $\mathrm{D}_{2}$ & 6707.90232 & $3.689 \times 10^{7}$ \\
${ }^{7} \mathrm{Li}$ & $\mathrm{D}_{1}$ & 6707.89719 & $3.689 \times 10^{7}$ \\
${ }^{7} \mathrm{Li}$ & $\mathrm{D}_{2}$ & 6707.74416 & $3.689 \times 10^{7}$ \\
\hline
\end{tabular}

Table 1: Wavelengths and Einstein $A$ coefficients for the $\mathrm{D}$ line transitions of $\mathrm{Li}$ isotopes.

\subsection{The diagonalization procedure}

The non-zero matrix elements of the total Hamiltonian defined in Equation (7) are of the form given by Equations (3.61a) and (3.61b) of LL04. Following Landi Degl'Innocenti (1978) we write a program to diagonalize the total Hamiltonian. The numerical diagonalization is performed using the Givens-Householder method. We obtain the eigenvalues in terms of the energy shifts from the parent $L$ state and the eigenvectors in terms of the $C$-coefficients. By making use of these energy shifts, we determine the energies of the $L_{a}=0$ and $L_{b}=1$ terms. Since $J$ is not a good quantum number in the PB regime, we cannot use either $\mathrm{D}_{1}$ or $\mathrm{D}_{2}$ wavelengths. For the atomic system we have considered, the line center wavelengths correspond to the transitions ${ }^{7} L_{a}=0 \rightarrow{ }^{7} L_{b}=1$ 


\begin{tabular}{|c|c|cc|cccc|}
\hline$J_{a} \backslash J_{b}$ & & $1 / 2$ & & $3 / 2$ & & & \\
\hline & $\mu_{a} \backslash \mu_{b}$ & $-1 / 2$ & $+1 / 2$ & $-3 / 2$ & $-1 / 2$ & $+1 / 2$ & $+3 / 2$ \\
\hline $1 / 2$ & $-1 / 2$ & $\pi^{\prime}$ & $\sigma_{\mathrm{b}}^{\prime}$ & $\sigma_{\mathrm{r}}$ & $\pi$ & $\sigma_{\mathrm{b}}$ & NA \\
& $+1 / 2$ & $\sigma_{\mathrm{r}}^{\prime}$ & $\pi^{\prime}$ & $\mathrm{NA}$ & $\sigma_{\mathrm{r}}$ & $\pi$ & $\sigma_{\mathrm{b}}$ \\
\hline
\end{tabular}

Table 2: The list of transitions between the magnetic substates of the upper and the lower $J$ states. NA - Not Allowed. The magnetic components of the two isotopes are distinguished in the following by their atomic masses indicated in the superscripts.

and ${ }^{6} L_{a}=0 \rightarrow{ }^{6} L_{b}=1$, which are, respectively, $6707.79517 \AA$ and $6707.95333 \AA$.

In the presence of a magnetic field, the degeneracy of the magnetic substates is lifted and the spectral lines split into magnetic components. It is possible to obtain the magnetic shifts and strengths of these components by making use of the $C$-coefficients and the energy eigenvalues. The normalized strengths of the transitions which connect the magnetic substates of the lower term $\left(L_{a} S\right)$ with those of the upper term $\left(L_{b} S\right)$ are given by

$$
\begin{aligned}
& \mathcal{S}_{q}^{j_{a} \mu_{a}, j_{b} \mu_{b}}=\alpha \sum_{J_{a} J_{a^{\prime}} J_{b} J_{b^{\prime}}} \frac{3}{2 S+1} \\
& \times C_{J_{a}}^{j_{a}}\left(L_{a} S, \mu_{a}\right) C_{J_{a^{\prime}}}^{j_{a}}\left(L_{a} S, \mu_{a}\right) \\
& \times C_{J_{b}}^{j_{b}}\left(L_{b} S, \mu_{b}\right) C_{J_{b^{\prime}}}^{j_{b}}\left(L_{b} S, \mu_{b}\right)\left(2 L_{a}+1\right) \\
& \times \sqrt{\left(2 J_{a}+1\right)\left(2 J_{a^{\prime}}+1\right)\left(2 J_{b}+1\right)\left(2 J_{b^{\prime}}+1\right)} \\
& \times\left(\begin{array}{ccc}
J_{b} & J_{a} & 1 \\
-\mu_{b} & \mu_{a} & -q
\end{array}\right)\left(\begin{array}{ccc}
J_{b^{\prime}} & J_{a^{\prime}} & 1 \\
-\mu_{b} & \mu_{a} & -q
\end{array}\right) \\
& \times\left\{\begin{array}{ccc}
L_{a} & L_{b} & 1 \\
J_{b} & J_{a} & S
\end{array}\right\}\left\{\begin{array}{ccc}
L_{a} & L_{b} & 1 \\
J_{b^{\prime}} & J_{a^{\prime}} & S
\end{array}\right\} .
\end{aligned}
$$

Here $\alpha$ represents the percentage abundance of the isotope. The magnetic shifts are given by

$$
\Delta_{\mu_{a} \mu_{b}}^{j_{a} j_{b}}=\frac{E_{j_{b}}\left(L_{b} S, \mu_{b}\right)-E_{j_{a}}\left(L_{a} S, \mu_{a}\right)}{h}+\delta E_{\text {iso }},
$$

where $E_{j}$ are the energy eigenvalues. $h$ is the Planck's constant. $\delta E_{\text {iso }}$ is the isotopic shift measured with respect to the reference isotope ${ }^{7} \mathrm{Li}$. Note that $\delta E_{\text {iso }}$ is zero for the reference isotope ${ }^{7} \mathrm{Li} . \Delta \mathrm{s}$ are given in frequency units.

Figure1 1 shows the behavior of the energy eigenvalues of the magnetic substates belonging to upper $J$ states as a function of the magnetic field strength $B$. As described in LL04, we define a

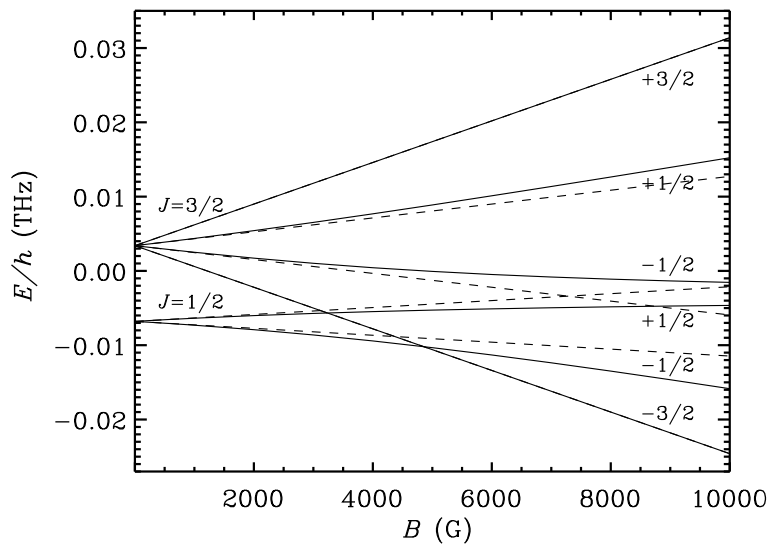

Fig. 1. - Level crossing between the magnetic substates belonging to the ${ }^{2} \mathrm{P}$ term of ${ }^{7} \mathrm{Li}$ isotope in the presence of a magnetic field. A comparison between the splittings produced by including (solid lines) and neglecting (dashed lines) $\mathrm{PBE}$. The ${ }^{2} \mathrm{P}$ term of ${ }^{6} \mathrm{Li}$ isotope gives a similar diagram except for the isotopic shift.

parameter $\gamma$ as

$$
\gamma=\frac{\mu_{0} B}{\zeta}
$$

which is a ratio of the magnetic energy to the fine structure energy. The energy eigenvalues diverge linearly with increase in the magnetic field strength for $\gamma \ll 1$. This regime is called the linear Zeeman regime. For intermediate values of $\gamma$, non-linearity sets in and the eigenvalues start to cross. This regime is called the incomplete PB regime. For $\gamma \gg 1$ the eigenvalues again vary linearly with $B$ and this regime is called the complete PB regime. For the atomic system considered we see two level-crossings. The values of $\gamma$ and $B$ for which these crossings occur are listed in Table 3 .

The solid lines in Figure 1 are computed taking the non-zero non-diagonal elements of the total 

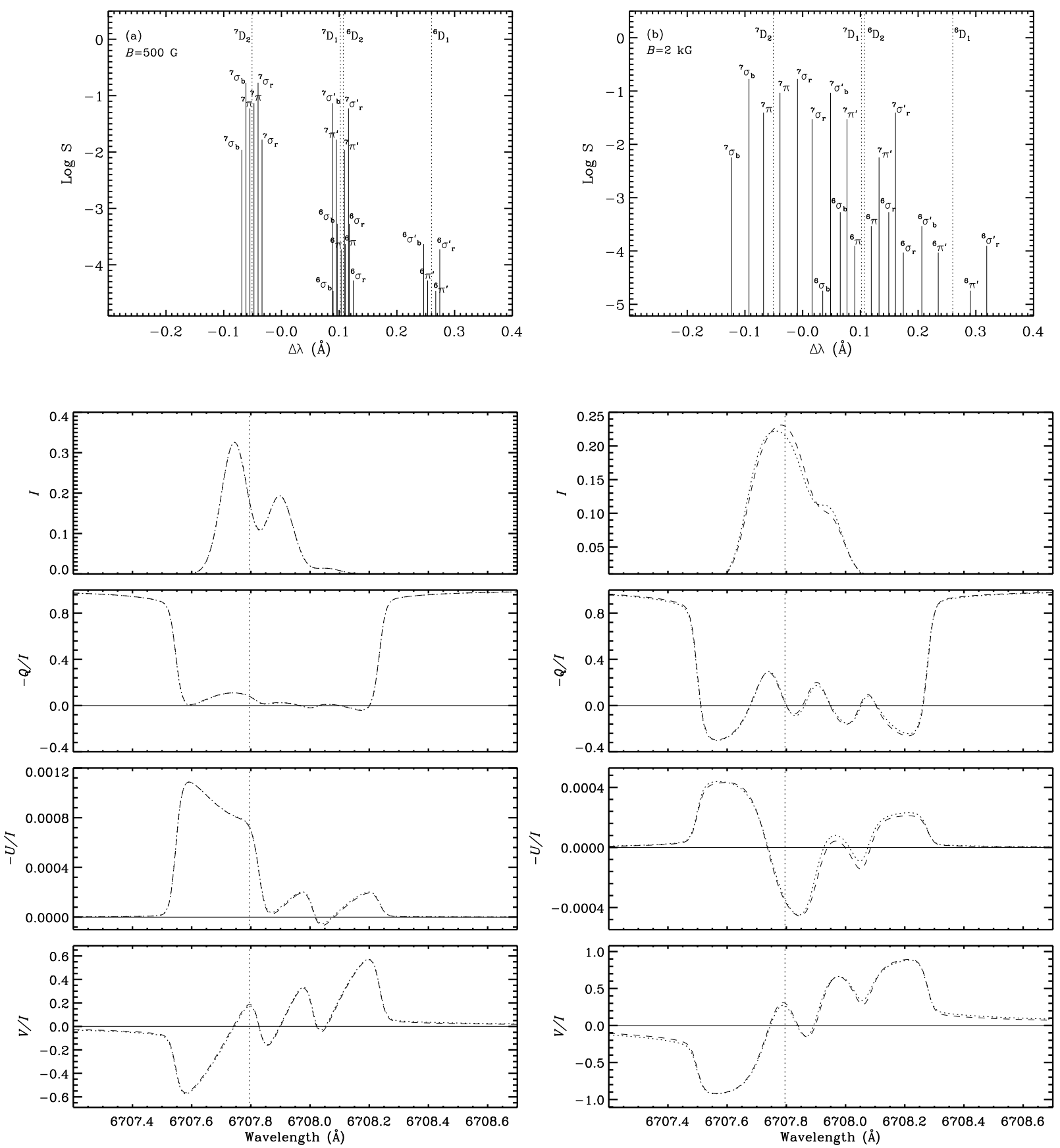

Fig. 2.- Top panels refer to the line splitting diagrams (line strength $\mathcal{S}$ vs. wavelength shift). The other panels show the comparison of the Stokes profiles computed using the PB-FS code (dashed lines) with the Zeeman-FS code (dotted lines). The two columns correspond to different field strengths as indicated in the line splitting diagrams. The vertical dotted lines in the line splitting diagrams indicate the positions of the ${ }^{7} \mathrm{Li}$ and ${ }^{6} \mathrm{Li} \mathrm{D}$ lines. The vertical dotted lines in other panels correspond to the line center wavelength of the $L=0 \rightarrow 1 \rightarrow 0$ transition in the reference isotope ${ }^{7} \mathrm{Li}$. The orientation of the magnetic field is given by $\left(\theta_{B}, \phi_{B}\right)=\left(90^{\circ}, 45^{\circ}\right)$. 

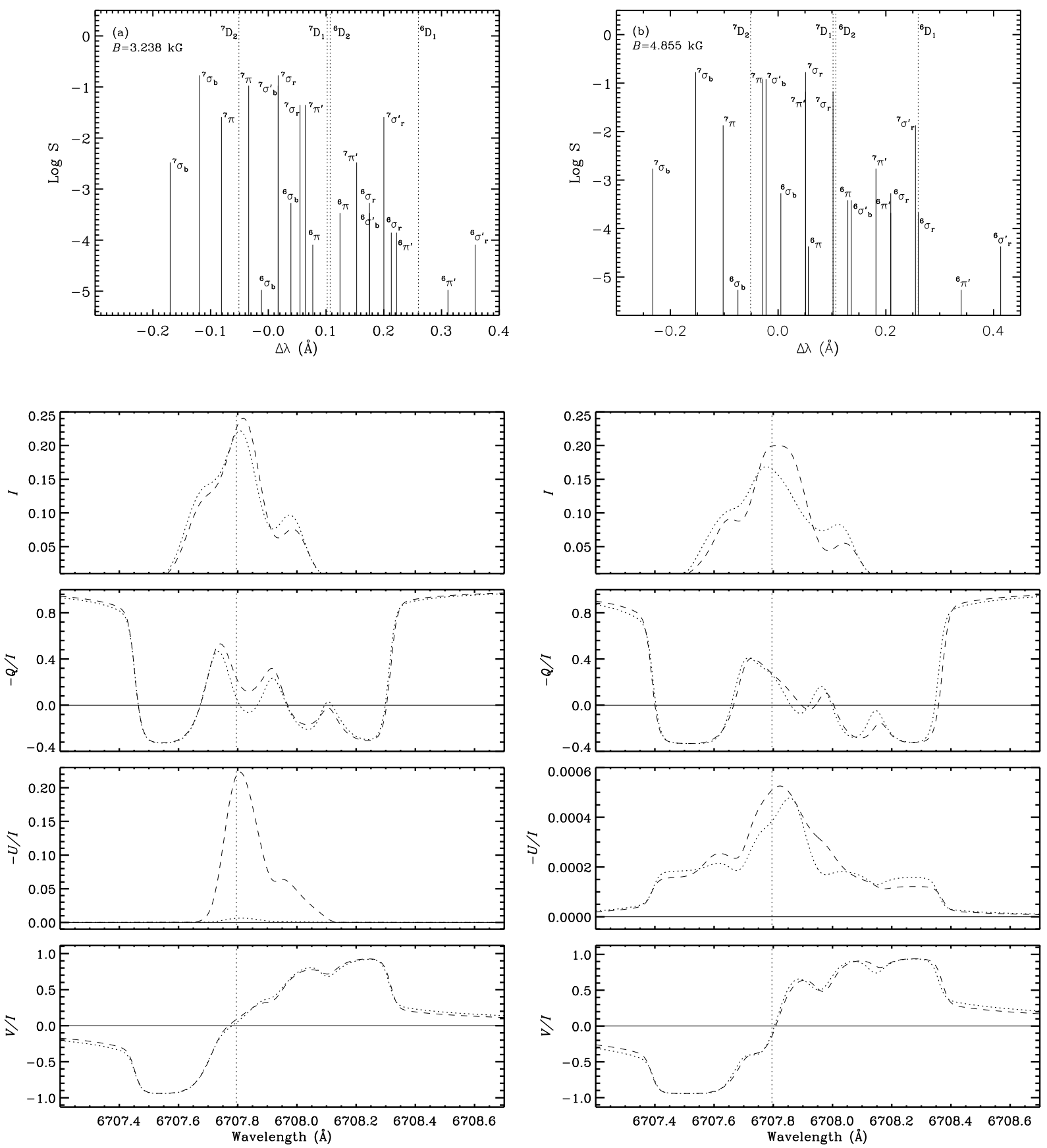

Fig. 3.- Same as Figure 2 except for field strengths. 
Hamiltonian (see Equation (3.61b) of LL04) into account while the dashed lines are computed by neglecting them. This means that, for the dashed lines, the splitting produced by the magnetic field is just given by the expression $\mu_{0} B g_{J_{b}} \mu_{b}$ (where $g_{J_{b}}$ is the Landé factor). We can clearly see the differences that PBE makes to the energy eigenvalues, from this diagram. The substates with $\mu_{b}=+3 / 2$ and $-3 / 2$ show the same behavior irrespective of whether PBE is included or not. This is because the contribution from the nondiagonal elements for these $\mu_{b} \mathrm{~s}$ are zero, as these $\mu_{b}$ s can be assigned to only $J_{b}=3 / 2$ state. For the other magnetic substates the splitting becomes nonlinear because of the contribution from the non-diagonal elements to the total splitting caused by the magnetic field. In particular we notice that the magnetic substates which cross in the case of Zeeman effect avoid crossing one another in the case of PBE. For example, the $\mu_{b^{\prime}}=+1 / 2$ belonging to $J_{b^{\prime}}=1 / 2$ and $\mu_{b}=-1 / 2$ belonging to $J_{b}=3 / 2$ cross at $B \sim 7.3 \mathrm{kG}$ when magnetic splittings are computed using the Zeeman effect. On the other hand, when PBE is included to compute the magnetic splitting, these substates do not cross. This is known as avoided crossing (also known as anti-level-crossing). As a consequence of this we find that the polarization in the asymptotic limit of $B \rightarrow \infty$ is larger than that when $B \rightarrow 0$. See Bommier (1980) and LL04 for more details on this effect.

\begin{tabular}{|c|c|cc|}
\hline$\mu_{b^{\prime}}$ & $\mu_{b}$ & $\gamma$ & $B(\mathrm{kG})$ \\
\hline$-3 / 2$ & $1 / 2$ & 0.667 & 3.238 \\
$-3 / 2$ & $-1 / 2$ & 1.0 & 4.855 \\
\hline
\end{tabular}

Table 3: The values of $\gamma$ and approximate values of $B$ at level-crossings for the ${ }^{2} \mathrm{P}$ term of ${ }^{7} \mathrm{Li}$ for which the level-crossing diagram shown in Figure 1 is made. The same table holds good for the ${ }^{2} \mathrm{P}$ term of ${ }^{6} \mathrm{Li}$.

\subsection{Comparison of Stokes profiles com- puted using linear Zeeman and PB ef- fects}

In the linear Zeeman regime, the RM presented in Section 3 reduces to Equation (25) of Smitha et al. (2011). In order to show the effects of level-crossing we compare the results of our code which programs Equation (11) (hereafter called as PB-FS code) with the results of Smitha et al. (2011) (hereafter called as Zeeman-FS code). This comparison is shown in Figures 2 and 3 . The Stokes profiles from the two codes match very well up to $500 \mathrm{G}$ for which $\gamma=0.1029$. According to the classification scheme discussed in the previous section, we are still in the linear Zeeman regime for this field strength. For field strengths larger than $500 \mathrm{G}$, the differences start to appear as we already enter the non-linear regime in which the linear Zeeman approximation (ZeemanFS code) breaks down. The separation between the magnetic components (which increases with an increase in $B$ ) is no longer given by $\mu_{0} B g_{J_{b}} \mu_{b}$. Hence there is a difference in the line center positions of the magnetic components computed from the two codes. These small differences are clearly seen in intensity $(I)$ profiles (see right panels of Figure 2). For level crossing field strengths (3.238 $\mathrm{kG}$ and $4.855 \mathrm{kG}$ ) the Stokes profiles computed from the Zeeman-FS and PB-FS codes differ drastically. The Zeeman-FS code therefore does not cover all the field strength ranges that we can expect on the Sun.

\subsection{Stokes profiles in the $\mathrm{PB}$ regime}

By making use of the strengths and shifts of the PB components obtained from the diagonalization code, we have made line splitting diagrams where the log of the $\mathrm{PB}$ component strengths $(\mathcal{S})$ given in Equation (12) are plotted against their wavelength shifts from the line center computed using Equation (13). These are shown in Figures 2 and 3 along with the Stokes profiles for different values of $B$. The zero on the x-axis of the line splitting diagram corresponds to the line center wavelength of the $L=0 \rightarrow 1$ transition in ${ }^{7} \mathrm{Li}$.

For $B=500 \mathrm{G}$ (linear regime), the magnetic components are separated into two bunches of 6 and 4 each, in both the isotopes. The magnetic components of the ${ }^{7} \mathrm{D}_{1}$ and ${ }^{6} \mathrm{D}_{2}$ lines superpose due to their proximity in wavelength. The splitting is more or less symmetric about the line centers of the $\mathrm{D}_{1}$ and $\mathrm{D}_{2}$ lines but the strengths of the components vary depending on the values of the magnetic quantum numbers $\mu_{a}$ and $\mu_{b}$ (see the left top panel of Figure 22). The same is reflected in the intensity profiles. The three peaks seen in intensity correspond to the three bunches of magnetic components with the amplitudes of 
the peaks being proportional to the relative abundances of the two isotopes. The $Q / I$ and $U / I$ profiles show typical signatures of Hanle effect especially at the position of the ${ }^{7} \mathrm{D}_{2}$ line, namely a depolarization of the $Q / I$ with respect to the nonmagnetic value (0.428 in the line core) and a generation of $U / I$ signal. The ${ }^{7} \mathrm{D}_{1}$ and ${ }^{6} \mathrm{D}_{1}$ lines are non-polarizing and hence are unaffected by Hanle effect. The ${ }^{6} \mathrm{D}_{2}$ line, although affected by Hanle effect, produces insignificant signatures due to its small abundance. In spite of these we see $U / I$ peaks at the positions corresponding to $\left({ }^{7} \mathrm{D}_{1},{ }^{6} \mathrm{D}_{2}\right)$ and ${ }^{6} \mathrm{D}_{1}$, the origin of which is not clear. They are possibly due to the interference between the D lines. Note however that these $U / I$ signatures are about three orders of magnitude smaller than the corresponding $Q / I$ signatures and are therefore much too weak to be observable. The $V / I$ arises due to the longitudinal component of the magnetic field.

For $B=2 \mathrm{kG}$ (see panels (b) of Figure 2), the components are well separated and their strengths change because of the dependence on the $C$ coefficients which vary with $B$. The components cannot be resolved in intensity as their Doppler width is larger than the separation between them. The decrease in the intensity is due to an increased separation between the magnetic components with increasing magnetic field strength. In $Q / I$, a three-lobed Zeeman like pattern is seen, $U / I$ is very small because of the geometry. The $V / I$ profiles become broader as expected. The $\sigma_{\mathrm{b}}$ components show opposite behavior to those of $\sigma_{\mathrm{r}}$ again as expected. Positive peaks appear at the positions of the $\sigma_{\mathrm{r}}$ components while negative peaks occur at the positions corresponding to $\sigma_{\mathrm{b}}$.

For $B=3.238 \mathrm{kG}$ (see panels (a) of Figure 3) at which the first level crossing occurs, we see the interference between the $\sigma_{\mathrm{r}}$ and $\sigma_{\mathrm{b}}^{\prime}$ components in the two isotopes. Their positions overlap as can be seen from the line splitting diagram. Interestingly, at these positions we see a generation of $U / I$ signal due to interference between the magnetic substates (Hanle effect). The $V / I$ signals of the $\mathrm{D}$ lines overlap giving rise to a broader profile.

For $B=4.855 \mathrm{kG}$ (see panels (b) of Figure 3) at which the second level crossing occurs, there is interference between the $\sigma_{\mathrm{r}}$ and $\pi^{\prime}$ components in the two isotopes. The $U / I$ signal is generated due to the Hanle effect.
In Figure 4 we show the Stokes profiles obtained from the PB-FS code in the presence of a weakly polarized background continuum. The contribution from the continuum is included in the same way as in Stenflo (1998). For the sake of clarity, we recall his Equations (58) and (61):

$$
\begin{gathered}
I^{\prime} / I_{c}^{\prime}=1-\beta+\frac{a}{I+a} \beta \\
p^{\prime}=\frac{I}{I+a} p+\frac{a}{I+a} b
\end{gathered}
$$

In the above equations $I$ and $p$ are the intensity and fractional polarization given by $-Q / I,-U / I$ and $V / I$ in the absence of the continuum. The corresponding quantities in the presence of the continuum are $I^{\prime}$ and $p^{\prime}$. The limb-darkening parameter, $\beta$, and the continuum strength parameter, $a$, are chosen as 0.5 and 0.1 , respectively. Such a large value of $a$ is chosen to make the Stokes profiles resemble closely with the profiles presented for the non-magnetic case in Belluzzi et al. (2009). The continuum polarization parameter, $b$, is chosen as 0.01 for $Q$ and 0 for $U$ and $V$. With this choice we obtain profile shapes of the kind that we expect in the Sun's spectrum. In particular our non-magnetic $p_{Q}^{\prime}$ profile (solid line) resembles the shape of the corresponding profile observed with ZIMPOL (Stenflo 2011). The intensity profiles appear as broad absorption lines. The fractional linear polarization $p_{Q}^{\prime}\left(=-Q^{\prime} / I^{\prime}\right)$ approaches the continuum polarization value $(b=0.01)$ away from the line center. The $p_{U}^{\prime}\left(=-U^{\prime} / I^{\prime}\right)$ and $p_{V}^{\prime}$ $\left(=V^{\prime} / I^{\prime}\right)$ profiles retain their overall shape compared to the pure line case without continuum, although their amplitudes decrease because of the contribution from the continuum strength parameter $a$ to $I^{\prime}$. As can be seen from the figure, the shape of the $p_{Q}^{\prime}$ profile for the zero field case (solid line) compares well with the corresponding profiles presented in Belluzzi et al. (2009). Note that since the Stokes profiles are computed here for a single scattering event, only the shape and not the amplitude is comparable to the corresponding profiles presented by Belluzzi et al. (2009).

\subsection{Polarization diagrams}

The geometry considered for the results presented in this section is shown in Figure 5 The plots of $Q / I$ vs. $U / I$ (polarization diagrams) are 

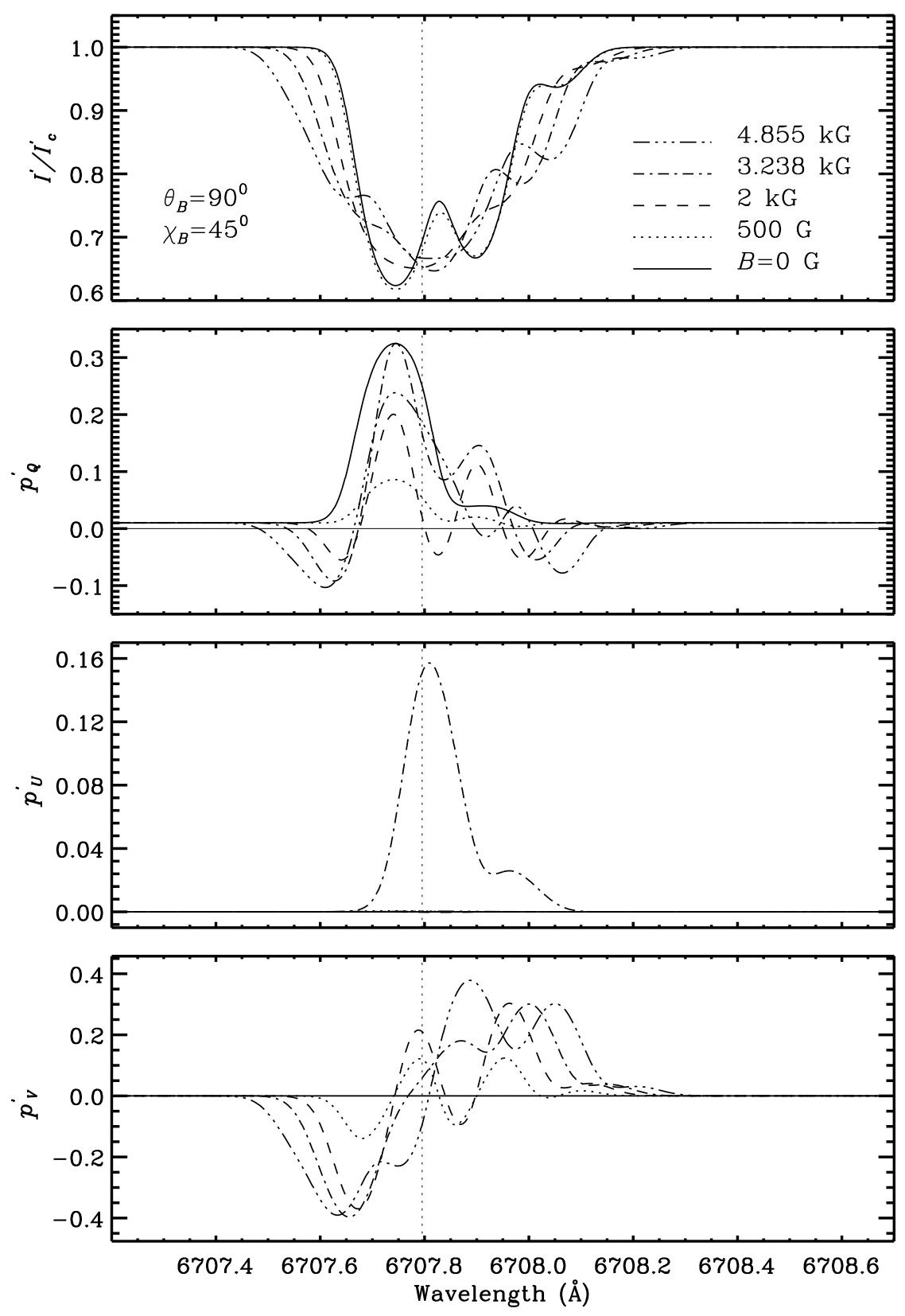

Fig. 4.- Stokes profiles computed using PB-FS code by including the contribution from the polarized continuum. The line types and the orientation of the magnetic field are indicated in the intensity panel. The vertical dotted lines in all the panels correspond to the line center wavelength of the $L=0 \rightarrow 1 \rightarrow 0$ transition in the reference isotope ${ }^{7} \mathrm{Li}$. See text for more details. 
shown for the line center wavelengths of the $\mathrm{Li} \mathrm{D}$ lines in Figure 6. For the geometry considered, only the level-crossings with $|\Delta \mu|=2$ are effective. Therefore, in the following, we will only see the effects due to the first level-crossing at 3.238 $\mathrm{kG}$.

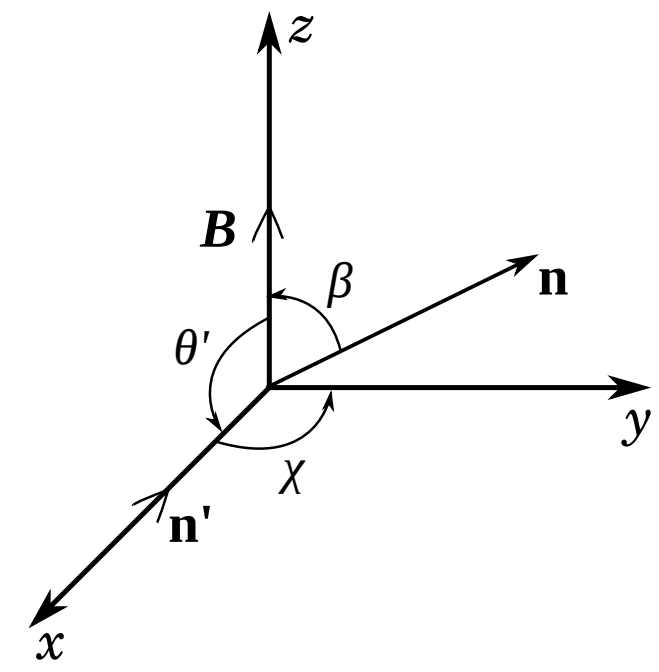

Fig. 5.- Geometry considered for computing the polarization diagrams shown in Figure 6. $\beta$ is the angle between the magnetic field vector and the scattered beam. The incident radiation is characterized by $\left(\theta^{\prime}, \chi^{\prime}\right)=\left(90^{\circ}, 0^{\circ}\right)$ and the scattered radiation by $(\theta, \chi)=\left(\beta, 90^{\circ}\right)$. The magnetic field inclination, $\theta_{B}=0^{\circ}$ and its azimuth, $\chi_{B}=0^{\circ}$ (magnetic reference frame).

At the ${ }^{7} \mathrm{D}_{2}$ line center $(\lambda=6707.74416 \AA)$ we see a decrease in $Q / I$ up to a few hundred gauss (Hanle regime), with an initial increase followed by a decrease in $U / I$ (see Figure 6 6 ). This is due to the Hanle effect which operates in the line core. As we approach the level-crossing field strength $(B=3.238 \mathrm{kG})$, we see an increase in the value of $Q / I$ and formation of a loop. Indeed the level-crossing occurs over a narrow range of field strengths around $3.238 \mathrm{kG}$. Within this narrow range if the field strength is varied by organizing a fine grid, we get a strong variation in $Q / I$ and $U / I$, producing the polarization diagram shown in Figure 6 . This behavior is generic to all the polarization diagrams shown in Figure 6. Further discussion on the formation of loops around the level-crossing field strengths can be found in LL04.
Basically at the level-crossing field strengths, the coherence between the overlapping magnetic substates increases, resulting in the scattered $Q / I$ tending towards the non-magnetic value. For $\mathrm{kG}$ fields, $U / I$ becomes zero because of the geometry considered. For fields stronger than $10 \mathrm{kG}$ (see Figure 6b), $Q / I$ arises due to Rayleigh scattering in strong magnetic fields, as discussed by Bommier (1997, Section 6, Figure 14). The author states that in this case, for the geometry considered (magnetic field along the line of sight), and for a $90^{\circ}$ scattering, only the $\sigma$ components are scattered, if the atomic system considered is a normal Zeeman triplet $(J=0 \rightarrow 1 \rightarrow 0)$. Incidentally we notice the same behavior in the case of $L=0 \rightarrow 1 \rightarrow 0$ transition (which is not a normal Zeeman triplet). It is interesting to note that the $\pi$ components are not scattered in this case also. The $Q / I$ changes sign and increases for fields up to $100 \mathrm{kG}$. At the ${ }^{6} \mathrm{D}_{2}$ line center $(\lambda=6707.90232 \AA$; see Figure 6r) the $Q / I$ for zero magnetic field case is approximately ten times smaller than the $Q / I$ at the ${ }^{7} \mathrm{D}_{2}$ line center. This is due to the relatively small abundance of ${ }^{6} \mathrm{Li}$. Due to an increased separation between the magnetic components with the field strength, the $Q / I$ value decreases. As in the case of ${ }^{7} D_{2}$ we notice the formation of a loop near the level-crossing field strength. When the field strength is increased beyond $10 \mathrm{kG}$, we again notice Rayleigh scattering in strong magnetic fields (not shown in the figure).

The ${ }^{6} \mathrm{D}_{1}$ line $(\lambda=6708.05534 \AA$; see Figure $6 \mathrm{~d})$ is intrinsically unpolarizable as it has $W_{2}=0$. Therefore the polarization remains zero until the level-crossing field strength $(B=3.238 \mathrm{kG})$ is reached. A further increase in the field strength results in the formation of a loop and Rayleigh scattering as already described for the ${ }^{7} \mathrm{D}_{2}$ and ${ }^{6} \mathrm{D}_{2}$ line center positions.

\section{Conclusions}

The theory of Hanle effect in a two-term atom was developed by LL04 assuming a flat-spectrum for the incident unpolarized radiation using the density matrix formalism. Though this theory is applicable to the entire range of magnetic fields, it does not take into account the effects of PRD. Smitha et al. (2011) included the effects of PRD by taking the redistribution matrix approach but 

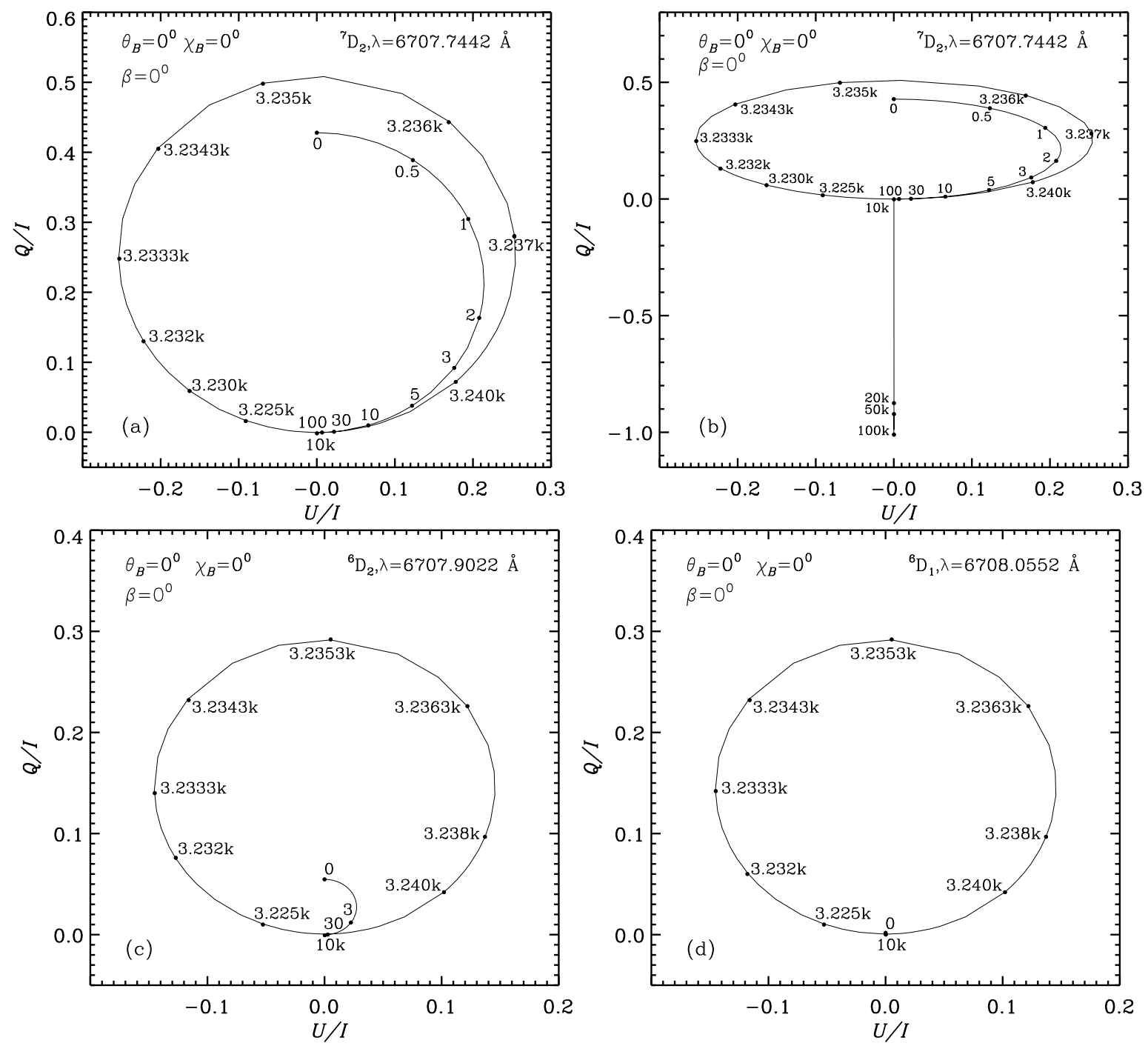

Fig. 6.- Polarization diagrams computed using the PB-FS code at the line center wavelengths of the ${ }^{7} \mathrm{Li}$ and ${ }^{6} \mathrm{Li} \mathrm{D}$ lines. The curves are marked by the values of the magnetic field strength $B$ in $\mathrm{G}$. A ' $\mathrm{k}$ ' means a factor of 1000 . 
their treatment was limited to the linear Zeeman regime. In the present paper we have generalized the approach of Smitha et al. (2011) for magnetic fields of arbitrary strengths to include the Paschen-Back regime. However, our treatment ignores the effects of collisions. Further the lower term is assumed to be unpolarized. The frequency dependence of the incident radiation field is taken into account in our theory which is essentially a relaxation of the flat-spectrum approximation of LL04. This enables us to properly calculate the scattered Stokes profile shapes which was otherwise not possible with the theory presented in LL04.

An example where the present theory has observable effects on the Sun is for the lithium $6708 \AA$ doublet. Since the fine structure splitting in this line system is small $(0.15 \AA)$, Paschen-Back effects in scattering polarization become prominent for magnetic fields that occur on the Sun. We have therefore tested our theory by applying it to this lithium doublet and demonstrated the correctness of the formalism by reproducing available benchmarks. We have explored the properties of the redistribution matrix for the single scattering case, and clarified the effects of Rayleigh scattering in strong fields when the magnetic field is along the line of sight. This has given us an overview of the behavior of the polarization effects that can occur as a result of PRD in the Paschen-Back regime.

We acknowledge the use of HYDRA cluster at the Indian Institute of Astrophysics for computations in this work.

\section{REFERENCES}

Asensio Ramos, A., \& Trujillo Bueno, J. 2006, ApJ, 636, 548

Belluzzi, L., Landi Degl'Innocenti, E., \& Trujillo Bueno, J. 2009, ApJ, 705, 218

Berdyugina, S. V., Braun, P. A., Fluri, D. M., \& Solanki, S. K. 2005, A\&A, 444, 947

Berdyugina, S. V., Fluri, D. M., \& Solanki, S. K. 2006a, ASPC, 358, 329

Berdyugina, S. V., Fluri, D. M., Ramelli, R., Bianda, M., Gisler, D., \& Stenflo, J. O. 2006b, ApJ, 649, L49

Bommier, V. 1980, A\&A, 87, 109
Bommier, V. 1997, A\&A, 328, 726

Casini, R., \& Manso Sainz, R. 2005, ApJ, 624, 1025

Landi Degl'Innocenti, E. 1978, A\&AS, 33, 157

Landi Degl'Innocenti, E., Landolfi, M. 2004, Polarization in Spectral Lines (Dordrecht: Kluwer) (LL04)

Shapiro, A. I., Fluri, D. M., Berdyugina, S. V., \& Stenflo, J. O. 2006, ASPC, 358, 311

Shapiro, A. I., Fluri, D. M., Berdyugina, S. V., \& Stenflo, J. O. 2007, A\&A, 461, 339

Smitha, H. N., Sampoorna, M., Nagendra, K. N., \& Stenflo, J. O. 2011, ApJ, 733, 4

Smitha, H. N., Nagendra, K. N., Sampoorna, M., \& Stenflo, J. O. 2013, JQSRT, 115, 46

Sowmya, K., Nagendra, K. N., Stenflo, J. O., \& Sampoorna, M. 2014, ApJ, 786, 150

Stenflo, J. O. 1980, A\&A, 84, 68

Stenflo, J. O. 1994, Sol. Magnetic Fields (Kluwer Academic Publishers)

Stenflo, J. O. 1997, A\&A, 324, 344

Stenflo, J. O. 1998, A\&A, 338, 301

Stenflo, J. O. 2011, in Solar Polarization 6, ASP Conference Series 437, eds. J. R. Kuhn, D. M. Harrington, H. Lin, S. V. Berdyugina, J. Trujillo-Bueno, S. L. Keil, \& T. Rimmele (San Francisco: ASP), 3

This 2-column preprint was prepared with the AAS LATEX macros $\mathrm{v} 5.2$. 